\title{
Metabolomic determinants of metabolic risk in Mexican adolescents
}

\author{
Wei Perng ${ }^{1,2}$, Emily C. Hector ${ }^{3}$, Peter X.K. Song ${ }^{3}$, Martha Maria Tellez Rojo ${ }^{4}$, \\ Sasha Raskind ${ }^{5}$, Maureen Kachman ${ }^{5}$, Alejandra Cantoral ${ }^{4}$, \\ Charles F. Burant ${ }^{1,5,6}$, and Karen E. Peterson ${ }^{1,7}$ \\ ${ }^{1}$ Department of Nutritional Sciences, University of Michigan School of Public Health, Ann Arbor, MI, \\ USA. \\ ${ }^{2}$ Department of Epidemiology, University of Michigan School of Public Health, Ann Arbor, MI, USA. \\ ${ }^{3}$ Department of Biostatistics, University of Michigan School of Public Health, Ann Arbor, MI, USA. \\ ${ }^{4}$ Center for Research on Nutrition and Health, National Institute of Public Health, Cuernavaca, Morelos, \\ México. \\ ${ }^{5}$ Michigan Regional Comprehensive Metabolomics Resource Core, University of Michigan Medical \\ School, Ann Arbor, MI, USA. \\ ${ }^{6}$ Center for Computational Medicine and Bioinformatics, University of Michigan Medical School, Ann \\ Arbor, MI, USA. \\ ${ }^{7}$ Center for Human Growth and Development, University of Michigan, Ann Arbor, MI, USA.
}

Running title: Metabolomics and metabolic risk in adolescents

Corresponding author:

Wei Perng (perngwei@umich.edu)

1415 Washington Heights, Room 1860 SPH 1

Ann Arbor, MI 48109-2029

Conflict of interest disclosure: None of the authors have any conflict of interest.

\#Words in abstract (max 200): 200

\#Words in manuscript body (max 3500): 3491

\# Tables: 5

\# Figures: 0

\# Supplemental files: 1 supplemental table

Key words: metabolic syndrome, adolescents, metabolomics, simulation and extrapolation algorithm

This is the author manuscript accepted for publication and has undergone full peer review but has not been through the copyediting, typesetting, pagination and proofreading process, which may lead to differences between this version and the Version record. Please cite this article as doi:10.1002/ oby.21926. 


\section{WHAT IS ALREADY KNOWN?}

- Disturbances in certain metabolic pathways, such as fatty acid and amino acid metabolism, are associated with obesity and obesity-related diseases in school-age children and adults.

- Identifying metabolites associated with metabolic risk during sensitive periods of development, such as adolescence, could provide insight on pathophysiology of metabolic diseases and enhance preventive efforts.

\section{WHAT THIS STUDY ADDS:}

- Using untargeted metabolomics data, we identified several metabolites on lipid, amino acid, and DNA pathways that were associated with a metabolic syndrome risk score (MetRisk zscore) in a cohort of 238 Mexican youth 8 to 14 years of age.

- In girls, diacylglycerol (DG) 16:0/16:0, 1,3-dielaidin, myo-inositol, and urate corresponded with higher MetRisk z-score, whereas $\mathrm{N}$-acetylglycine, thymine, and dodecenedioic acid were associated with lower MetRisk z-score.

- In boys, DG 16:0/16:0, tyrosine, and 5'-methylthioadenosine were each positively associated with MetRisk z-score. 


\section{ABSTRACT}

Objective: To identify metabolites associated with metabolic risk, separately by sex, in Mexican adolescents.

Methods: We carried out untargeted metabolomic profiling on fasting serum of 238 youth age 814 years, and identified metabolites associated with a metabolic syndrome risk z-score (MetRisk z-score), separately for boys and girls using the simulation and extrapolation (SIMEX) algorithm. We examined associations of each metabolite with MetRisk z-score using linear regression models that accounted for maternal education, child's age, and pubertal status.

Results: Of the 938 features identified in metabolomics analysis, 7 named compounds (of 27 identified metabolites) were associated with MetRisk z-score in girls, and 3 named compounds (of 14 identified) were associated with MetRisk z-score in boys. In girls, diacylglycerol (DG) 16:0/16:0, 1,3-dielaidin, myo-inositol, and urate corresponded with higher MetRisk z-score, whereas $\mathrm{N}$-acetylglycine, thymine, and dodecenedioic acid were associated with lower MetRisk z-score. For example, each z-score increment in DG 16:0/16:0 corresponded with 0.60 (0.47, 0.74). In boys, we found positive associations of DG 16:0/16:0, tyrosine, and 5'methylthioadenosine with MetRisk z-score.

Conclusions: Metabolites on lipid, amino acid, and carbohydrate metabolism pathways are associated with metabolic risk in girls. Compounds on lipid and DNA pathways correspond with metabolic risk in boys. 


\section{INTRODUCTION}

Metabolomics, the systematic study of low-molecular-weight compounds in biological tissues, is a powerful tool to study disease onset, severity, and progression. In adults, metabolomics analyses of serum and plasma have provided novel insights into biomarkers and etiology of metabolic disorders - the most commonly-studied being type 2 diabetes (1). Given that the pathophysiological processes underlying metabolic disease development (e.g. adiposity, insulin resistance, dyslipidemia, hypertension) begin as early as 2 to 4 years of age (2-4) and may be established for life by the end of adolescence $(5,6)$, there is need to elucidate the relationship between circulating metabolites and conventional metabolic biomarkers earlier in life.

Few metabolomics analyses have been carried out in youth, and the majority of published studies sought to replicate findings from adult populations by focusing on associations of specific compounds on amino acid (7-10) and fatty acid pathways $(7,10)$ with weight status and/or glycemia. However, although adult studies $(11,12)$ and two metabolomics analyses in school-age children $(13,14)$ found that higher circulating branched chain amino acids (BCAAs) correlated with insulin resistance and/or type 2 diabetes risk, animal models (15) and data from a clinical cohort adolescents with and without diabetes $(7,8)$ revealed that these metabolites actually corresponded with better glycemia and enhanced mitochondrial function. A potential explanation for the discrepancy is that physiological responses to metabolic disturbances may be fundamentally different during the pubertal transition, as it is a time of rapid change and hormonal fluctuation Therefore, approaches aimed at validating findings from adults or prepubertal children may fail to identify relevant metabolic pathways in youth undergoing puberty.

Here, our primary aim was to take a data-driven approach to identify metabolites associated with a cluster of metabolic syndrome risk factors during puberty - a life stage 
characterized by rapid growth and development that may also be a sensitive period for development of excess adiposity and metabolic risk (16). As a secondary aim, we examined associations of BCAAs (leucine, isoleucine, valine) with the metabolic syndrome risk factors given their relevance to metabolic risk in previous metabolomics studies in youth.

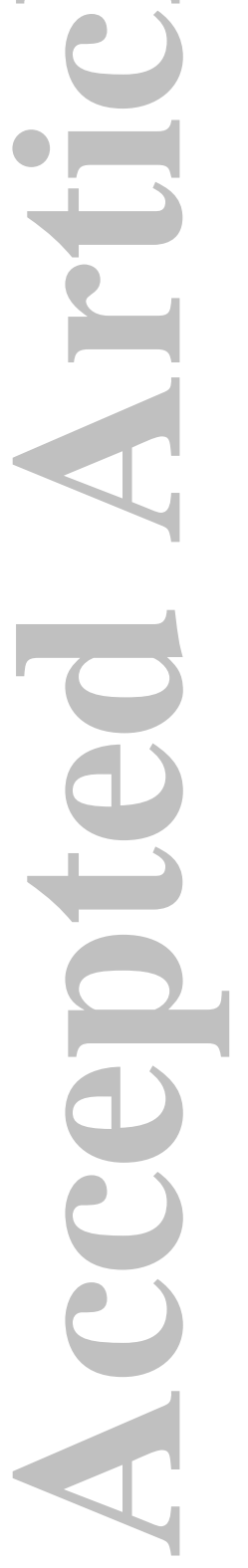




\section{METHODS}

\section{Study population}

This study included participants of the Early Life Exposure in Mexico to Environmental Toxicants (ELEMENT) Project, a cohort of pregnant women and their offspring in Mexico City, Mexico (17). In 2010, we re-contacted a subset of the offspring $(n=250)$ who were then 8-14 years of age based on availability of archived prenatal biospecimens to participate in follow-up studies. At research visits that took place in 2010 (the "peripubertal" visit), the participants provided an 8-hour fasting blood sample and participated in anthropometric assessment. Our study sample included 238 children with data on anthropometry, metabolic biomarkers, and adequate serum volume for metabolomics analyses. The institutional review boards of the Mexico National Institute of Public Health and the University of Michigan approved research protocols.

\section{Untargeted metabolomics profiling}

The Michigan Regional Comprehensive Metabolomics Resource Core $\left(\mathrm{MRC}^{2}\right)$ quantified metabolites from fasting serum collected at the peripubertal visit on an untargeted platform that utilized liquid chromatography and mass spectrometry (LC/MS). The procedure yielded 9,303 features. After removal of redundant compounds and those with $>70 \%$ of values missing, the final data set comprised 938 unique chemical features, 332 of which were "known" compounds e.g., those whose spectral peaks, fragmentation patterns, and retention times matched with compounds within $\mathrm{MRC}^{2}$, s chemical library. While we considered all metabolites in the statistical analysis, our interpretation of results focuses on known compounds. Prior to formal analyses, we removed batch effects, imputed values below the detection using the K-nearest 
neighbor algorithm $(\mathrm{K}=5)$ using the IMPUTE package in $\mathrm{R}$, and standardized each metabolite as a z-score using a rank-based inverse normal transformation.

\section{Conventional biomarkers}

The primary outcome of interest was a metabolic syndrome risk z-score (MetRisk zscore) calculated as the average of 5 internally-standardized z-scores for waist circumference, fasting glucose, fasting C-peptide (as a surrogate for fasting insulin (18)), the triglycerides to high-density lipoprotein (HDL) ratio, and the average of systolic (SBP) and diastolic blood pressure (DBP). This score is a modification (e.g., use of C-peptide rather than insulin) of a score proposed by Viitasalo et al. in a study that examined correlations among the metabolic biomarkers in children and adults, and established its association with incident type 2 diabetes and cardiovascular mortality (19).

Waist circumference. Research staff measured waist circumference at the level of the umbilicus o the nearest $0.1 \mathrm{~cm}$ using a non-stretchable measuring tape (QM2000; QuickMedical). Fasting glucose, C-peptide, and lipids. Using 8-hour fasting blood, we measured serum glucose enzymatically. Serum C-peptide was quantified using an automated chemiluminescence immunoassay (Immulite 1000, Siemens Medical Solutions). These biomarkers provide a measure of glycemic control as fasting glucose is an indicator of glucose metabolism and a diabetes screening tool, and C-peptide is a marker of insulin function that is secreted in quantities equal to insulin from pancreatic $\beta$-cells (18). We quantified serum triglycerides and HDL using a biochemical analyzer (Cobas Mira Plus, Roche Diagnostics).

Blood pressure. Research staff measured SBP and DBP in duplicate to the nearest $\mathrm{mmHg}$ in the seated position (BPTru monitors; Coquitlam, BC, Canada). Because the intra-class correlation 
(ICC) between the measurements were high $\left(\mathrm{ICC}_{\mathrm{SBP}}=0.95 ; \mathrm{ICC}_{\mathrm{DBP}=0.89}\right.$ ), we used the average in the analysis.

Supplemental Table 1 shows correlations among the MetRisk z-score components.

\section{Covariates}

At enrollment, mothers reported on age, reproductive history, and lifestyle and sociodemographic characteristics. A pediatrician assessed each child to determine Tanner stage on a scale of 1 (no development) to 5 (full development) for testicle, breast, and pubic hair.

\section{Data analysis}

Step 1-Bivariate analysis. We examined the distribution of MetRisk z-score across categories of background characteristics to identify potential confounders.

Step 2 -Identification of relevant metabolites. To identify metabolites associated with metabolic risk, we examined sex-specific associations (given the differences in tempo of maturation during adolescence $(20))$ of individual metabolites $(n=938)$ with MetRisk z-score, while also adjusting for maternal education and pubertal status, using the simulation extrapolation (SIMEX) algorithm (SIMEX package in R). Our rationale for using SIMEX for the initial "first pass" to identify relevant metabolites is to correct for additive measurement error (e.g., residual batch effects; unmeasured variation in laboratory methods) in the assessment of metabolite concentrations. In the first step, the SIMEX algorithm simulates multiple datasets with varying degrees of measurement error in the metabolite of interest in order to estimate a range of coefficients that plausibly represent the error-free relationship between the metabolite and MetRisk z-score. Next, SIMEX derives an estimate for a covariate that represents the impact of the unknown source(s) of error based on the simulated data and extrapolates an error-corrected estimate for the association of interest. As a result, SIMEX estimates for regression parameters 
are larger in magnitude and more precise than those derived from standard regression. For confirmatory purposes, we also quantified associations of individual metabolites with MetRisk zscore using standard linear regression. To reduce the likelihood of false positive associations, we only retained metabolites for further analyses if they were associated with MetRisk z-score at a $P$-value of $<0.05 / 938$ (Bonferroni's correction).

Step 3 - Main analysis. We used multivariable linear regression to assess the impact of potential confounders to the relationship of the known metabolites identified in Step 2, as well as the BCAAs, with MetRisk z-score. We selected covariates based on our a priori knowledge and the bivariate analysis from Step 1. Because there is little known regarding metabolomics of pubertal status, a potential confounder and mediator to the relationship between metabolism and metabolic risk, we also examined crude associations of the metabolites of interest with pubertal status to ascertain its relevance as a covariate (Supplemental Table 2). In Model 1, we accounted for confounders: maternal education and child's age. In Model 2, we further adjusted for pubertal status. Using Model 2, we also investigated associations of all metabolites of interest with components of the z-score.

Unless otherwise stated, all statistical analyses were performed using SAS 9.4 (Cary, NC, USA). 


\section{RESULTS}

Median age of the participants was 10.0 years (range: $8.1,14.7$ ) and approximately half of the sample $(47.5 \% ; n=113)$ were boys. Mean \pm SD MetRisk z-score and the individual components of the score are displayed in Table $\mathbf{1 .}$

In bivariate analysis (Table 2), older children and those at more advanced pubertal stages exhibited higher metabolic risk. As compared to participants younger than 10 years of age, those 10-12 years and $>12$ years of age exhibited 0.20 (95\% CI: $0.03,0.38)$ and $0.36(0.16,0.55)$ units higher MetRisk z-score. In both boys and girls, we observed a positive relationship between all puberty indicators and MetRisk z-score.

Of the 938 features quantified in laboratory analyses, the SIMEX algorithm identified 27 compounds, 7 of which were known, in girls and 14 compounds, 3 of which were known, in boys that were associated with MetRisk z-score after Bonferroni's correction (Table 3). A comparison of the magnitude, direction, and significance of the estimates derived from standard linear regression vs. SIMEX showed that the latter yielded larger effect sizes $(\sim 1.5-2$ times the magnitude) and smaller $P$-values, which is expected given that the SIMEX procedure accounts for error in assessment of the metabolite concentrations.

After identification of relevant metabolites, we investigated associations ( $\beta[95 \% \mathrm{CI}])$ of each known compound with MetRisk z-score using multivariable linear regression (Table 4). In girls, we found positive relations of diacylglycerol (DG) 16:0/16:0, 1,3-dielaidin, myo-inositol, and urate with MetRisk z-score; and inverse associations with $\mathrm{N}$-acetylglycine, thymine, and dodecenedioic acid. Adjustment for maternal education and child's age (Model 1) followed by pubertal status (Model 2) did not change the results. For example, each 1 z-score increment in 
DG 16:0/16:0 corresponded with $0.63(0.49,0.76)$ units higher MetRisk z-score in the unadjusted model. Accounting for mother's education and child's age did not materially alter the estimate $(0.61[0.47,0.75])$, nor did further inclusion of pubertal status $(0.60[0.47,0.74])$.

Likewise, each $1 \mathrm{z}$-score increment in thymine was associated with $0.38(0.22,0.54)$ units lower MetRisk z-score in Model 1, and 0.39 (0.23, 0.55) units lower MetRisk z-score in Model 2. In boys, we detected positive associations of DG 16:0/16:0 (0.42 [0.27, 0.57]), tyrosine (0.37 [0.21, $0.53])$, and 5'-methylthioadenosine (MTA) $(0.30[0.15,0.46])$ with MetRisk z-score in unadjusted models. These associations persisted after multivariable adjustment (Table 4).

We also examined relationships of each metabolite with individual components of the MetRisk z-score: waist circumference, fasting glucose, fasting C-peptide, triglyceride:HDL ratio, and the average of SBP and DBP (Table 5). In girls, the four compounds positively associated with MetRisk z-score were also positively related to each z-score component. We observed the most consistent associations for C-peptide. For example, DG 16:0/16:0, 1,3-dielaidin, myoinositol, and urate corresponded with $0.4(0.2,0.6), 0.3(0.1,0.5), 0.4(0.2,0.6)$, and $0.3(0.0,0.5)$ ng/mL C-peptide, respectively. We noted similar trends of these compounds with waist circumference. Similarly, the three compounds inversely related to MetRisk z-score (thymine, dodecenedioic acid, and $\mathrm{N}$-acetylglycine) were inversely associated with the individual biomarkers - again, with the most robust associations observed for waist circumference and Cpeptide.

In boys, each 1 z-score increment in DG 16:0/16:0, tyrosine, and 5'-MTA was associated with $3.1(1.4,4.8), 2.7(0.9,4.4)$, and $3.8(2.2,5.4) \mathrm{cm}$ higher waist circumference, respectively. These metabolites were also consistently positively associated with C-peptide, and to a lesser extent, the triglyceride:HDL ratio and blood pressure. 
Table 6 shows associations of BCAAs with the metabolic biomarkers. In girls, valine corresponded with higher triglyceride:HDL ratio $(0.3[0.1,0.5])$. In boys, all three BCAAs were associated with higher waist circumference, fasting C-peptide, and MetRisk z-score (Table 6). For example, each $1 \mathrm{z}$-score increment in leucine corresponded with $3.0(1.4,4.5) \mathrm{cm}$ higher waist circumference, $0.3(0.2,0.5) \mathrm{ng} / \mathrm{mL}$ higher $\mathrm{C}$-peptide, and $0.13(0.03,0.24)$ units higher MetRisk z-score. We observed associations of similar direction and magnitude for isoleucine and valine.
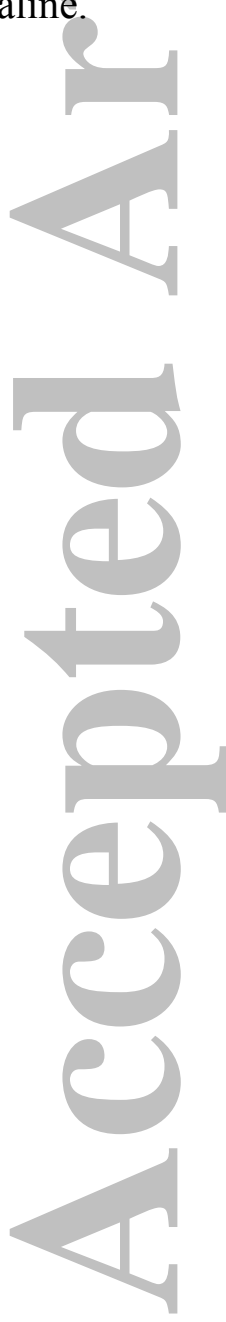


\section{DISCUSSION}

In this study of 238 Mexican youth 8-14 years of age, we employed a data-driven approach to identify metabolites associated with metabolic syndrome risk factors. Use of untargeted metabolomic profiling followed by measurement-error corrected regression models revealed associations of metabolites on lipid, amino acid, and carbohydrate metabolism pathways with metabolic risk in girls, whereas compounds on lipid and DNA pathways were related to metabolic risk in boys. As a secondary endpoint, we examined associations of BCAAs (leucine, isoleucine, valine), which have been previously implicated in metabolic risk in youth $(7-9,13,14)$, with the metabolic outcomes and found positive associations with metabolic risk in boys.

\section{Data-driven approach: girls}

In girls, we identified 7 named metabolites that were associated with MetRisk z-score. Of these compounds, four were positively related to metabolic risk: diacylglycerol 16:0/16:0, 1,3dielaidin, myo-inositol, and urate; and 3 were inversely associated with metabolic risk: thymine, dodecenedioic acid, and N-acetylglycine.

\section{Metabolites associated with higher MetRisk z-score}

Our finding that higher serum levels of diacylglycerol (DG) 16:0/16:0 - a glyceride composed of two palmitic acid side chains - corresponded with higher metabolic risk makes sense given that DG 16:0/16:0 is a major component of palm oil, a saturated vegetable oil commonly used in developing nations like Mexico that adversely impacts plasma lipid profile (21). Indeed, when we examined associations with components of MetRisk z-score, DG 16:0/16:0 was strongly associated with a larger triglyceride-to-HDL ratio, which has been 
identified as a discriminatory biomarker of metabolic syndrome in children (22). Another pathway through which DG 16:0/16:0 may influence metabolic risk is by inducing insulin resistance. In a study that compared muscle tissue DG composition of $n=6$ non-diabetic obese adults, $n=6$ type 2 diabetic adults, and $n=6$ endurance-trained athletes, Bergman et al. reported higher levels of three DG species, one of which was DG 16:0/16:0, in myocyte membranes of diabetic subjects than for the other two groups (23). Further, the authors observed a increasing monotonic relationship between the DGs and insulin resistance in all three groups (23) - a phenomenon that could be explained the capacity of DGs to activate protein kinase-C, an enzyme implicated in the pathogenesis of skeletal muscle insulin resistance (24). Accordingly, this compound was associated with higher fasting C-peptide, a surrogate marker of insulin (18), in girls.

We also found positive associations of 1,3-dielaidin, myo-inositol, and urate with MetRisk Z-score. Although there are no published studies relating 1,3-dielaidin - a glycerol with two monounsaturated fatty acid chains - to metabolic health, a few mechanisms could underlie our findings for the other two compounds. Myo-inositol plays an important role as a precursor for secondary cellular messengers such as inositol phosphates phosphatidylinositol and phosphatidylinositol phosphate-containing lipids (25). While myo-inositol occurs naturally in fruits, grains, beans, and nuts, it is also generated endogenously from D-glucose to augment cellular glucose uptake (26), a process that may be upregulated under conditions of hyperglycemia and/or insulin resistance (27) to encourage glucose utilization. This notion is supported by our finding that myo-inositol corresponded with higher fasting glucose and Cpeptide. 
We observed a positive association between urate and MetRisk z-score, which aligns with literature pointing towards elevated serum uric acid as a risk factor for cardio-metabolic disease. Proposed mechanisms include uric acid's ability to induce oxidative stress (28) and stimulate pro-inflammatory pathways (29), both of which interfere with metabolic homeostasis.

\section{Metabolites associated with lower MetRisk z-score}

In girls, we identified three metabolites that were inversely associated with metabolic risk: N-acetylglycine, dodecenedioic acid, and thymine. A few studies have identified Nacetylglycine as a correlate of weight status. In an untargeted metabolomics study of adults in three cohorts, Moore et al. found an inverse relationship between $\mathrm{N}$-acetylglycine and body mass index (BMI) under both fasting and non-fasting conditions (30). Similarly, in an untargeted analysis of fasting plasma from 803 Hispanic children, Butte et al. reported higher plasma Nacetylglycine of in non-obese compared to obese children (14). While the exact mechanism linking this compound to excess adiposity remains yet to be elucidated, a study in 73 elderly adults observed higher levels of $\mathrm{N}$-acetylglycine in the gut microbiome following ingestion of high-fiber foods (31). Given that dietary fiber intake is consistently associated with lower adiposity and metabolic risk in epidemiologic studies (32), mechanistic investigations are warranted to determine whether $\mathrm{N}$-acetylglycine is simply a marker of fiber metabolism, or if it imparts a direct protective effect against metabolic risk.

The inverse relationship between dodecenedioic acid and metabolic risk could transpire from its antioxidant properties. A recent in vitro study of human skin fibroblasts showed that administration of dodecenedioic acid decreased phospholipid perodixation (33), a process that is upregulated under conditions of hyperglycemia. 
Finally, our finding that higher serum concentrations of thymine corresponded with lower metabolic risk could stem from the physiological effects of its catabolite $\beta$-aminoisobutyrate (BAIBA), a compound released by skeletal muscle that enhances glucose tolerance (34).

\section{Data-driven approach: boys}

As with girls, DG 16:0/16:0 was positively associated with MetRisk z-score in boys, emphasizing the relevance of this compound to metabolic health regardless of sex. In addition, we also found that higher serum levels of tyrosine and 5'-MTA corresponded with greater metabolic risk among males.

Our finding of a positive association between tyrosine, a large neutral amino acid, and MetRisk z-score, as well as with adiposity (larger waist circumference) and worse glycemia (higher C-peptide) corroborates literature in both adults and children. Because tyrosine relies on the same protein (LAT1) as BCAAs for membrane transport, circulating tyrosine concentrations directly correlate with BCAAs. Tyrosine is often captured as part of the BCAA "metabolic signature" that is associated with obesity and insulin resistance in cross-sectional studies of adults (11) and children $(13,14)$, and with incident type 2 diabetes among young adults in the Framingham cohort (12). Of particular relevance to our findings are two recent investigations that identified tyrosine as a discriminatory marker of metabolic risk. In an analysis of 96 healthy Slovenian adults 25-49 years of age, Mohorko et al. (35) quantified 10 amino acids in fasting serum and categorized participants as having 0,1 , or 2 components of metabolic syndrome in order to determine whether certain amino acids had greater capacity than others to discriminate between the three levels of subclinical disease severity. The investigators found that tyrosine was higher among persons who met criteria for 1 vs. 0 components of metabolic syndrome, suggesting that it may serve as an early indicator of metabolic syndrome risk. Similarly, in a 
recent analysis of 80 obese European children, Hellmuth et al. (36) examined changes in glycemia and circulating levels of 163 metabolites related to amino acid metabolism before vs. after a 1-year weight-loss intervention and identified tyrosine as the only compound that correlated with insulin resistance at baseline, after the intervention, and with change in insulin resistance during the course of the study (36).

We also found a direct association between 5'-MTA with metabolic risk. While there have not been any studies specifically reporting on 5'-MTA with respect to metabolic health, 5'MTA is a byproduct of polyamine synthesis in DNA turnover cycles that increases with inflammation to modulate cellular stress (37). Considering that metabolic diseases are characterized by persistent chronic inflammation, it is plausible that the relationship between 5'MTA and the MetRisk z-score is driven by pro-inflammatory pathways.

\section{BCAAs and metabolic risk}

In addition to our data-driven approach, we also examined associations of BCAAs with the metabolic syndrome risk factors based on their relevance to metabolic health in previous studies of children and adolescents $(7-9,13,14)$. While the results were generally null for girls (with the exception of a positive relationship between valine and triglyceride:HDL), all three BCAAs corresponded with higher waist circumference, fasting C-peptide, and the MetRisk zscore in boys. These results corroborate the existing literature in youth regarding the relationship of BCAAs with excess adiposity and worse glycemia $(9,13,14)$, but warrant additional ivestigations in other populations to confirm the sex-specific nature of our findings. 
Strengths \& weaknesses

Strengths of this study include our data-driven approach, research-quality measures of body composition and metabolic biomarkers, data on important covariates including physicianassessed pubertal status, and use of the SIMEX algorithm to correct for unmeasured error in assessment of metabolite concentrations.

Our study has some limitations. First, the sample size was relatively small, although it is comparable to other untargeted metabolomics studies carried out in children/adolescents (13,

14). Second, the cross-sectional design impedes our ability to ascertain temporality. Third, assessment of the metabolites took place at a single time-point and thus, we are not able to make inference on metabolic flux. Finally, we cannot rule out the possibility of unmeasured confounding.

Conclusions

In this study of 238 Mexican youth 8-14 years of age, metabolites on lipid, amino acid, and carbohydrate metabolism pathways with metabolic risk in girls, and compounds on lipid, DNA, and BCAA pathways corresponded with greater metabolic risk in boys. The sex-specific nature of the associations between metabolites and metabolic risk have been observed in prepubertal obese children (38) and obese adolescents (39) in relation to insulin resistance, and with respect to metabolic syndrome in young adults (40), emphasizing the importance of considering sex differences in studies of metabolism. While our findings shed light on potential mechanisms underlying metabolic risk during adolescence, future studies are necessary to investigate associations of these compounds with prospective change in conventional metabolic biomarkers. 
ACKNOWLEDGEMENTS: We thank the American British Cowdry Hospital for their research facilities, and Alla Karnovsky, Jennifer LaBarre, and Lu Tang for help with metabolomics data processing.

FUNDING: This work was supported by the following grants: P01ES022844 from the National Institute for Environmental Health Sciences (NIEHS), and RD83543601 from the US

Environmental Protection Agency (US EPA), Michigan Nutrition Obesity Research Center (P30 DK089503) and Michigan Regional Comprehensive Metabolomics Resource Core (R24 DK097153) and the Robert C. and Veronica Atkins Foundation. This study was also supported by the National Institute of Public Health/Ministry of Health of Mexico. It contents are solely the responsibility of the grantee and do not necessarily represent the official views of the US EPA.

Further, the US EPA does not endorse the purchase any commercial products or services mentioned in the publication. 


\section{REFERENCES}

1. Guasch-Ferré M, Hruby A, Toledo E, Clish CB, Martínez-González MA, Salas-Salvadó J, et al. Metabolomics in Prediabetes and Diabetes: A Systematic Review and Meta-analysis. Diabetes Care. 2016;39(5):833-46.

2. Juhola J, Magnussen CG, Viikari JS, Kahonen M, Hutri-Kahonen N, Jula A, et al. Tracking of serum lipid levels, blood pressure, and body mass index from childhood to adulthood: the Cardiovascular Risk in Young Finns Study. J Pediatr. 2011;159(4):584-90.

3. Zinner SH, Rosner B, Oh W, Kass EH. Significance of blood pressure in infancy. Familial aggregation and predictive effect on later blood pressure. Hypertension. 1985;7(3 Pt $1): 411-6$.

4. Ibanez L, Ong K, Dunger DB, de Zegher F. Early development of adiposity and insulin resistance after catch-up weight gain in small-for-gestational-age children. J Clin Endocrinol Metab. 2006;91(6):2153-8.

5. Bao W, Srinivasan SR, Wattigney WA, Berenson GS. Persistence of multiple cardiovascular risk clustering related to syndrome $\mathrm{X}$ from childhood to young adulthood. The Bogalusa Heart Study. Arch Intern Med. 1994;154(16):1842-7.

6. Spalding KL, Arner E, Westermark PO, Bernard S, Buchholz BA, Bergmann O, et al. Dynamics of fat cell turnover in humans. Nature. 2008;453(7196):783-7.

7. Mihalik SJ, Michaliszyn SF, de las Heras J, Bacha F, Lee S, Chace DH, et al. Metabolomic profiling of fatty acid and amino acid metabolism in youth with obesity and type 2 diabetes: evidence for enhanced mitochondrial oxidation. Diabetes Care. 2012;35(3):605-11. 
8. Michaliszyn SF, Sjaarda LA, Mihalik SJ, Lee S, Bacha F, Chace DH, et al. Metabolomic profiling of amino acids and beta-cell function relative to insulin sensitivity in youth. J Clin Endocrinol Metab. 2012;97(11):E2119-24.

9. McCormack SE, Shaham O, McCarthy MA, Deik AA, Wang TJ, Gerszten RE, et al. Circulating branched-chain amino acid concentrations are associated with obesity and future insulin resistance in children and adolescents. Pediatric Obes. 2013;8(1):52-61.

10. Wahl S, Yu Z, Kleber M, Singmann P, Holzapfel C, He Y, et al. Childhood obesity is associated with changes in the serum metabolite profile. Obes Facts. 2012;5(5):660-70.

11. Newgard CB, An J, Bain JR, Muehlbauer MJ, Stevens RD, Lien LF, et al. A branchedchain amino acid-related metabolic signature that differentiates obese and lean humans and contributes to insulin resistance. Cell Metab. 2009;9(4):311-26.

12. Wang TJ, Larson MG, Vasan RS, Cheng S, Rhee EP, McCabe E, et al. Metabolite profiles and the risk of developing diabetes. Nat Med. 2011;17(4):448-53.

13. Perng W, Gillman MW, Fleisch AF, Michalek RD, Watkins SM, Isganaitis E, et al. Metabolomic profiles and childhood obesity. Obesity (Silver Spring, Md). 2014;22(12):2570-8. 14. Butte NF, Liu Y, Zakeri IF, Mohney RP, Mehta N, Voruganti VS, et al. Global metabolomic profiling targeting childhood obesity in the Hispanic population. Am J Clin Nutr. $2015 ; 102(2): 256-67$.

15. Lenaers E, De Feyter HM, Hoeks J, Schrauwen P, Schaart G, Nabben M, et al. Adaptations in mitochondrial function parallel, but fail to rescue, the transition to severe hyperglycemia and hyperinsulinemia: a study in Zucker diabetic fatty rats. Obesity (Silver Spring, Md). 2010;18(6):1100-7. 
16. Lee JM. Why young adults hold the key to assessing the obesity epidemic in children. Arch Pediatr Adolesc Med. 2008;162(7):682-7.

17. Hu H, Tellez-Rojo MM, Bellinger D, Smith D, Ettinger AS, Lamadrid-Figueroa H, et al. Fetal lead exposure at each stage of pregnancy as a predictor of infant mental development. Environ Health Perspect. 2006;114(11):1730-5.

18. Bonser AM, Garcia-Webb P. C-peptide measurement: methods and clinical utility. Crit Rev Clin Lab Sci. 1984;19(4):297-352.

19. Viitasalo A, Lakka TA, Laaksonen DE, Savonen K, Lakka HM, Hassinen M, et al. Validation of metabolic syndrome score by confirmatory factor analysis in children and adults and prediction of cardiometabolic outcomes in adults. Diabetologia. 2014;57(5):940-9.

20. Tanner JM. Chapter 5: Puberty. Foetus into Man. Cambridge: Harvard University Press; 1978. p. 58-74.

21. Vega-Lopez S, Ausman LM, Jalbert SM, Erkkila AT, Lichtenstein AH. Palm and partially hydrogenated soybean oils adversely alter lipoprotein profiles compared with soybean and canola oils in moderately hyperlipidemic subjects. Am J Clin Nutr. 2006;84(1):54-62.

22. Liang J, Fu J, Jiang Y, Dong G, Wang X, Wu W. TriGlycerides and high-density lipoprotein cholesterol ratio compared with homeostasis model assessment insulin resistance indexes in screening for metabolic syndrome in the chinese obese children: a cross section study. BMC Pediatr. 2015;15:138.

23. Bergman BC, Hunerdosse DM, Kerege A, Playdon MC, Perreault L. Localisation and composition of skeletal muscle diacylglycerol predicts insulin resistance in humans.

Diabetologia. 2012;55(4):1140-50. 
24. Griffin ME, Marcucci MJ, Cline GW, Bell K, Barucci N, Lee D, et al. Free fatty acidinduced insulin resistance is associated with activation of protein kinase $\mathrm{C}$ theta and alterations in the insulin signaling cascade. Diabetes. 1999;48(6):1270-4.

25. Downes CP, Macphee CH. myo-inositol metabolites as cellular signals. Eur J Biochem. 1990;193(1):1-18.

26. Ortmeyer HK. Dietary myoinositol results in lower urine glucose and in lower postprandial plasma glucose in obese insulin resistant rhesus monkeys. Obesity Res. 1996;4(6):569-75.

27. Ostlund RE, Jr., McGill JB, Herskowitz I, Kipnis DM, Santiago JV, Sherman WR. Dchiro-inositol metabolism in diabetes mellitus. Proc Natl Acad Sci U S A. 1993;90(21):9988-92. 28. Glantzounis GK, Tsimoyiannis EC, Kappas AM, Galaris DA. Uric acid and oxidative stress. Curr Pharm Des. 2005;11(32):4145-51.

29. Lu W, Xu Y, Shao X, Gao F, Li Y, Hu J, et al. Uric Acid Produces an Inflammatory Response through Activation of NF-kappaB in the Hypothalamus: Implications for the Pathogenesis of Metabolic Disorders. Scientific Rep. 2015;5:12144.

30. Moore SC, Matthews CE, Sampson JN, Stolzenberg-Solomon RZ, Zheng W, Cai Q, et al. Human metabolic correlates of body mass index. Metabolomics. 2014;10(2):259-69.

31. Lustgarten MS, Price LL, Chalé A, Fielding RA. Metabolites related to gut bacterial metabolism, peroxisome proliferator-activated receptor-alpha activation, and insulin sensitivity are associated with physical function in functionally-limited older adults. Aging Cell. 2014;13(5):918-25.

32. Lattimer JM, Haub MD. Effects of Dietary Fiber and Its Components on Metabolic Health. Nutrients. 2010;2(12):1266-89. 
33. Jabłońska-Trypuć A, Pankiewicz W, Czerpak R. Traumatic Acid Reduces Oxidative Stress and Enhances Collagen Biosynthesis in Cultured Human Skin Fibroblasts. Lipids. 2016;51(9):1021-35.

34. Shi CX, Zhao MX, Shu XD, Xiong XQ, Wang JJ, Gao XY, et al. beta-aminoisobutyric acid attenuates hepatic endoplasmic reticulum stress and glucose/lipid metabolic disturbance in mice with type 2 diabetes. Scientific Rep. 2016;6:21924.

35. Mohorko N, Petelin A, Jurdana M, Biolo G, Jenko-Praznikar Z. Elevated serum levels of cysteine and tyrosine: early biomarkers in asymptomatic adults at increased risk of developing metabolic syndrome. BioMed Res Int. 2015;2015:418681.

36. Hellmuth C, Kirchberg FF, Lass N, Harder U, Peissner W, Koletzko B, et al. Tyrosine Is Associated with Insulin Resistance in Longitudinal Metabolomic Profiling of Obese Children. J Diabetes Res 2016;2016:2108909.

37. Hevia H, Varela-Rey M, Corrales FJ, Berasain C, Martinez-Chantar ML, Latasa MU, et al. 5'-methylthioadenosine modulates the inflammatory response to endotoxin in mice and in rat hepatocytes. Hepatology. 2004;39(4):1088-98.

38. Mastrangelo A, Martos-Moreno GA, Garcia A, Barrios V, Ruperez FJ, Chowen JA, et al. Insulin resistance in prepubertal obese children correlates with sex-dependent early onset metabolomic alterations. Int J Obes. 2016;40(10):1494-502.

39. Newbern D, Gumus Balikcioglu P, Balikcioglu M, Bain J, Muehlbauer M, Stevens R, et al. Sex differences in biomarkers associated with insulin resistance in obese adolescents: metabolomic profiling and principal components analysis. J Clin Endocrinol Metab. 2014;99(12):4730-9. 
40. Rauschert S, Uhl O, Koletzko B, Mori TA, Beilin LJ, Oddy WH, et al. Sex differences in the association of phospholipids with components of the metabolic syndrome in young adults. Biol Sex Differ. 2017;8:10.
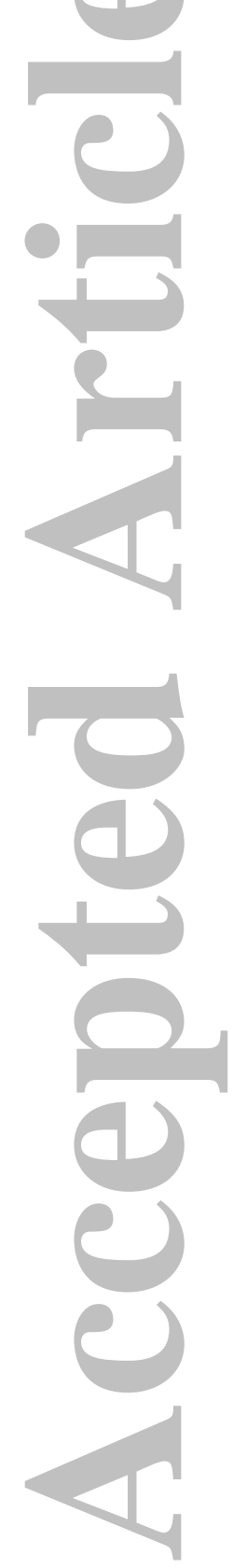
Table 1 Mean \pm SD of individual metabolic biomarkers and the metabolic risk z-score (MetRisk z-score) in 238 ELEMENT participants.

\begin{tabular}{lccc} 
& All & Girls & Boys \\
& $n=238$ & $n=125$ & $n=113$ \\
\cline { 2 - 4 } Waist circumference (cm) & $70.8 \pm 10.8$ & $71.6 \pm 11.5$ & $70.0 \pm 10.0$ \\
Fasting glucose (mg/dL) & $87.1 \pm 9.4$ & $86.2 \pm 10.6$ & $88.1 \pm 7.9$ \\
C-peptide (ng/dL) & $1.7 \pm 1.23$ & $1.9 \pm 1.3$ & $1.6 \pm 1.2$ \\
HDL (mg/dL) & $58.7 \pm 11.8$ & $57.7 \pm 11.7$ & $59.8 \pm 12.0$ \\
Triglycerides (mg/dL) & $87.8 \pm 44.2$ & $96.8 \pm 47.2$ & $77.9 \pm 38.4$ \\
SBP (mmHg) & $103 \pm 10$ & $101 \pm 10$ & $105 \pm 10$ \\
DBP (mmHg) & $66 \pm 7$ & $66 \pm 7$ & $66 \pm 7$ \\
MetRisk z-score $^{\mathrm{a}}$ & $0.00 \pm 0.62$ & $0.03 \pm 0.65$ & $-0.04 \pm 0.58$ \\
\hline
\end{tabular}

HDL: high-density lipoprotein, SBP: systolic blood pressure, DBP: diastolic blood pressure

a Calculated as the average of 5 internally-standardized z-scores for waist circumference, fasting glucose, C-peptide, triglycerides/HDL ratio, and the average of SBP and DBP.

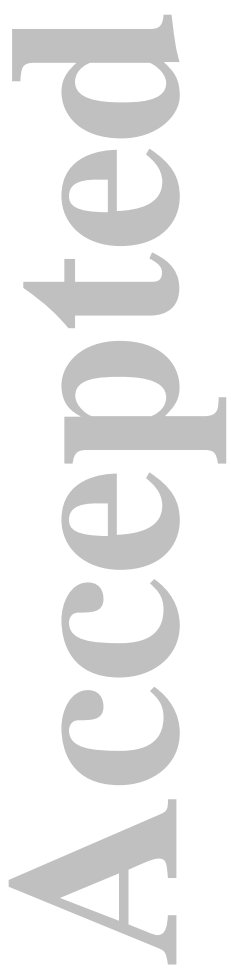


Table 2 Association of background characteristics with MetRisk z-score among 238 ELEMENT mother-child pairs.

\begin{tabular}{|c|c|c|c|c|c|}
\hline & \multirow{2}{*}{$N$} & \multicolumn{4}{|c|}{ MetRisk z-score } \\
\hline & & \multicolumn{3}{|c|}{ Mean \pm SD } & $P^{\mathbf{a}}$ \\
\hline \multicolumn{6}{|c|}{ Maternal characteristics at the time of enrollment } \\
\hline Age at enrollment & & & & & 0.62 \\
\hline $15-24$ years & 87 & 0.02 & \pm & 0.59 & \\
\hline $25-34$ years & 118 & -0.07 & \pm & 0.59 & \\
\hline $35-44$ years & 32 & 0.16 & \pm & 0.75 & \\
\hline Marital status & & & & & 0.23 \\
\hline Married or cohabiting & 212 & -0.02 & \pm & 0.62 & \\
\hline Single & 26 & 0.13 & \pm & 0.59 & \\
\hline Maternal education & & & & & 0.90 \\
\hline$<10$ years & 83 & -0.01 & \pm & 0.60 & \\
\hline 10 to 12 years & 119 & 0.00 & \pm & 0.62 & \\
\hline$\geq 13$ years & 34 & 0.00 & \pm & 0.65 & \\
\hline Parity & & & & & 0.40 \\
\hline 0 & 85 & 0.02 & & 0.58 & \\
\hline $1-2$ & 137 & 0.00 & & 0.62 & \\
\hline$\geq 3$ & 16 & -0.15 & & 0.77 & \\
\hline Smoking during pregnancy & & & & & 0.30 \\
\hline Yes & 233 & -0.27 & \pm & 0.51 & \\
\hline No & 5 & 0.01 & \pm & 0.62 & \\
\hline Delivery method & & & & & 0.29 \\
\hline C-section & 96 & 0.05 & \pm & 0.63 & \\
\hline Vaginal & 141 & -0.04 & \pm & 0.61 & \\
\hline Child characteristics at th & visit & & & & \\
\hline Sex & & & & & 0.39 \\
\hline Male & 113 & -0.04 & \pm & 0.58 & \\
\hline Female & 125 & 0.03 & \pm & 0.65 & \\
\hline Child's age & & & & & 0.0002 \\
\hline$<10$ years & 119 & -0.14 & \pm & 0.63 & \\
\hline 10 to 12 years & 68 & 0.07 & \pm & 0.56 & \\
\hline$>12$ years & 51 & 0.22 & \pm & 0.59 & \\
\hline Male puberty indicators ${ }^{b}$ & & & & & \\
\hline Pubic hair & & & & & 0.01 \\
\hline Tanner Stage 1 & 90 & -0.05 & \pm & 0.60 & \\
\hline Tanner Stage 2 & 15 & 0.18 & \pm & 0.62 & \\
\hline Tanner Stage 3-5 & 5 & 0.58 & \pm & 0.74 & \\
\hline Testicle development & & & & & 0.04 \\
\hline Tanner Stage 1 & 54 & -0.15 & \pm & 0.60 & \\
\hline Tanner Stage 2 & 40 & 0.18 & \pm & 0.64 & \\
\hline Tanner Stage 3 & 10 & -0.10 & \pm & 0.47 & \\
\hline Tanner Stage 4-5 & 5 & 0.36 & \pm & 0.91 & \\
\hline
\end{tabular}


Table 2 (continued) Association of background characteristics with MetRisk zscore among 238 ELEMENT mother-child pairs.

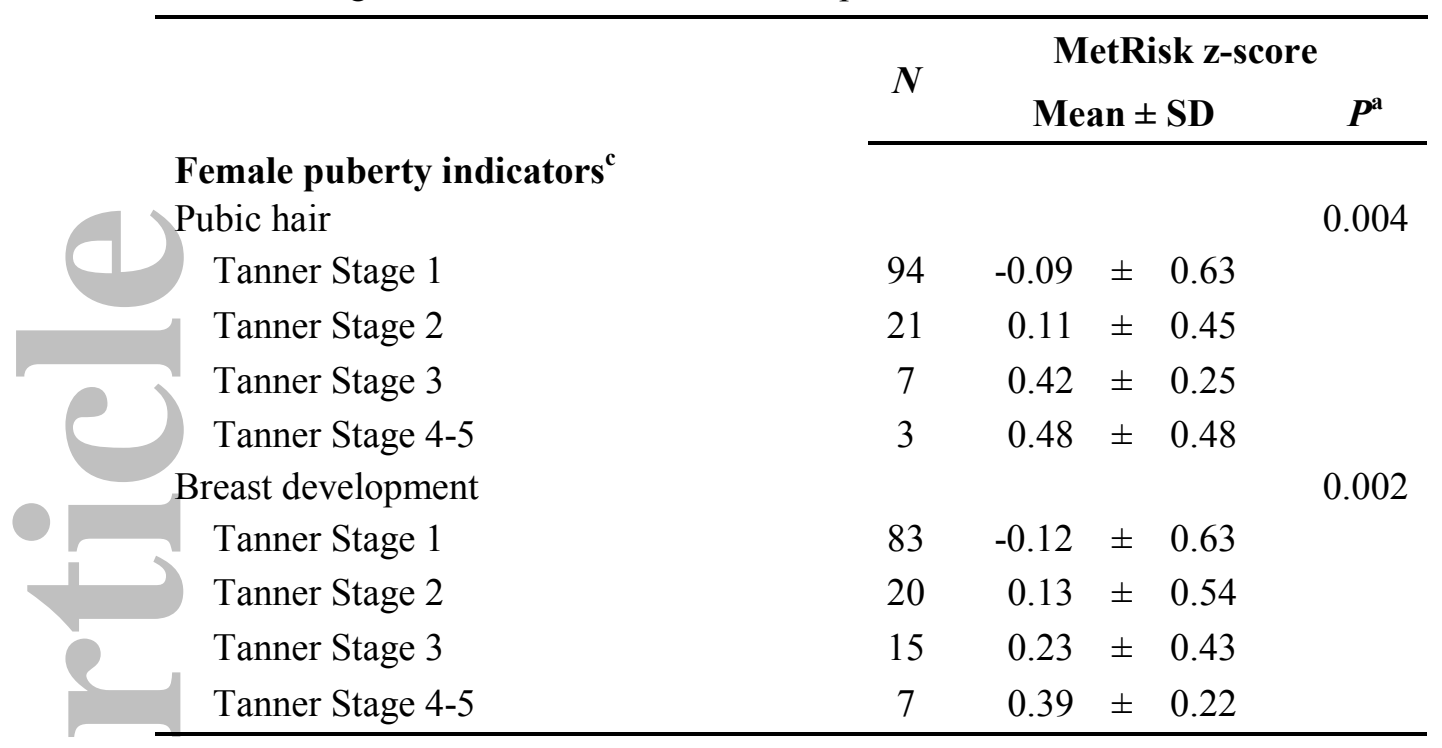

a Represents a test for linear trend where an ordinal indicator is entered into the model as continuous variable, with the exception of binary variables (Wald test).

b Tanner stages were combined due to small sample sizes for pubic hair Stages 3-5

(Stage 3: $n=4$; Stage 4: $n=1$, Stage 5: $n=0$ ); for testicle development Stages 4-5 (Stage 4: $n=5$; Stage $5: n=0$ ).

c Tanner stages were combined due to small sample sizes for pubic hair (Stage 4: $n=2$, Stage 5: $n=1$ ) and testicle development (Stage 4: $n=7$; Stage 5: $n=0$ ). 
Table 3 Association of metabolite concentrations with MetRisk z-score among 125 girls and 113 boys using standard linear regression and SIMEX.

Girls $(n=125)$

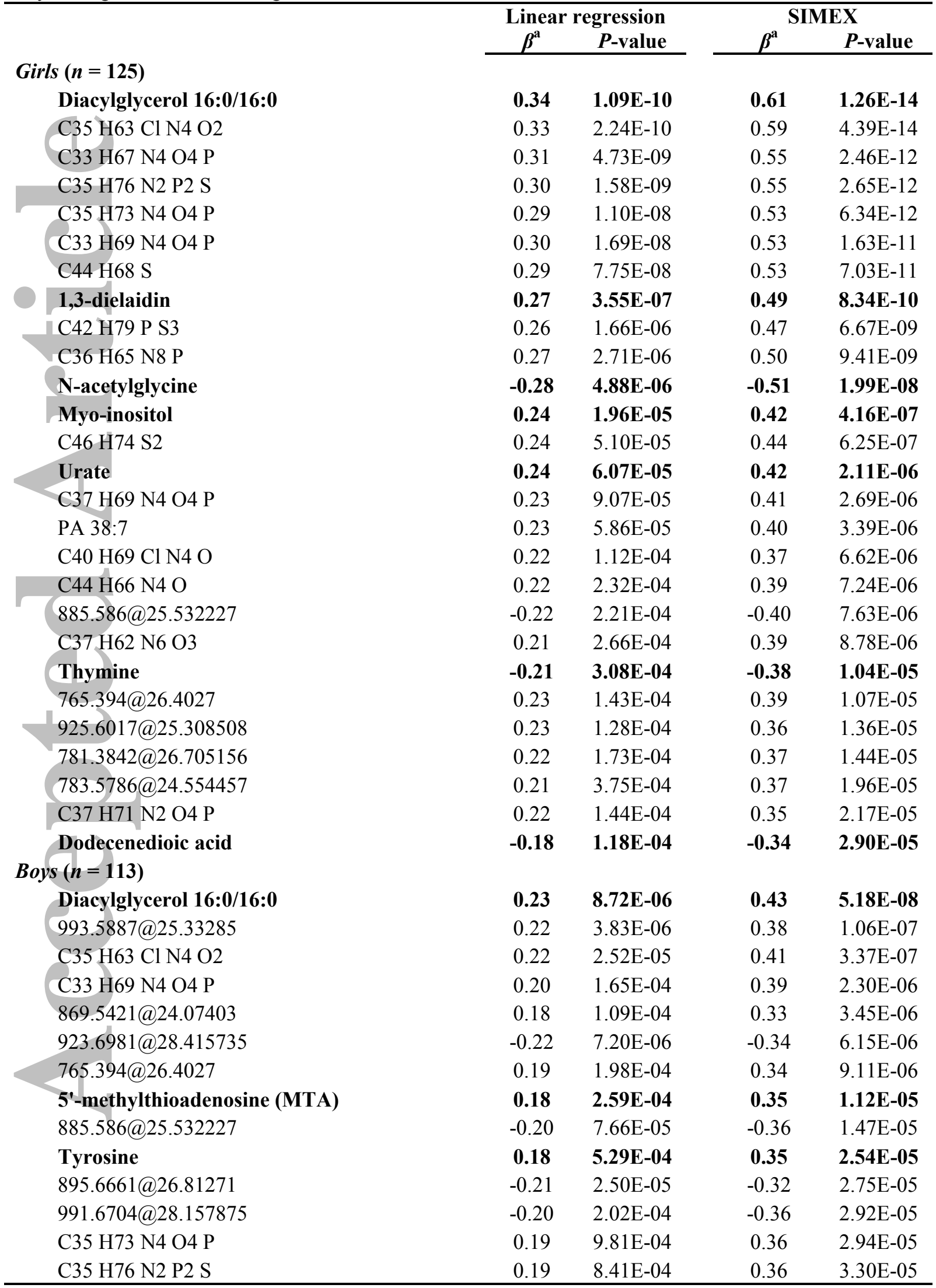

a Estimates are adjusted for mother's education level and child's age.

b Bolded estimates indicate statistical significance after Bonferroni's correction ( $P$-value $<5.33 \mathrm{E}-05)$ 
Table 4 Sex-specific associations of individual metabolites with MetRisk z-score among 238 ELEMENT adolescents.

\section{Girls $(n=125)$}

Diacylglycerol 16:0/16:0

1,3-dielaidin

Myo-inositol

Urate

Thymine

Dodecenedioic acid

$\mathrm{N}$-acetylglycine

ß (95\% CI) MetRisk z-score

per 1 unit increment in metabolite z-score

Unadjusted

Model 1

Model 2

Boys $(n=113)$

Diacylglycerol 16:0/16:0

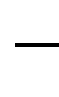

Tyrosine

$0.42(0.27,0.57)$

$0.43(0.29,0.58)$

$0.42(0.28,0.56)$

5'-methylthioadenosine (MTA)

$0.37(0.21,0.53)$

$0.35(0.19,0.50)$

$0.33(0.17,0.48)$

$0.30(0.15,0.46)$

$0.35(0.20,0.49)$

$0.32(0.18,0.46)$

Model 1: Adjusted for maternal education level and child's age.

Model 2: Model $1+$ pubertal status.

Bolded estimates indicate statistical significance at $P<0.05$. 


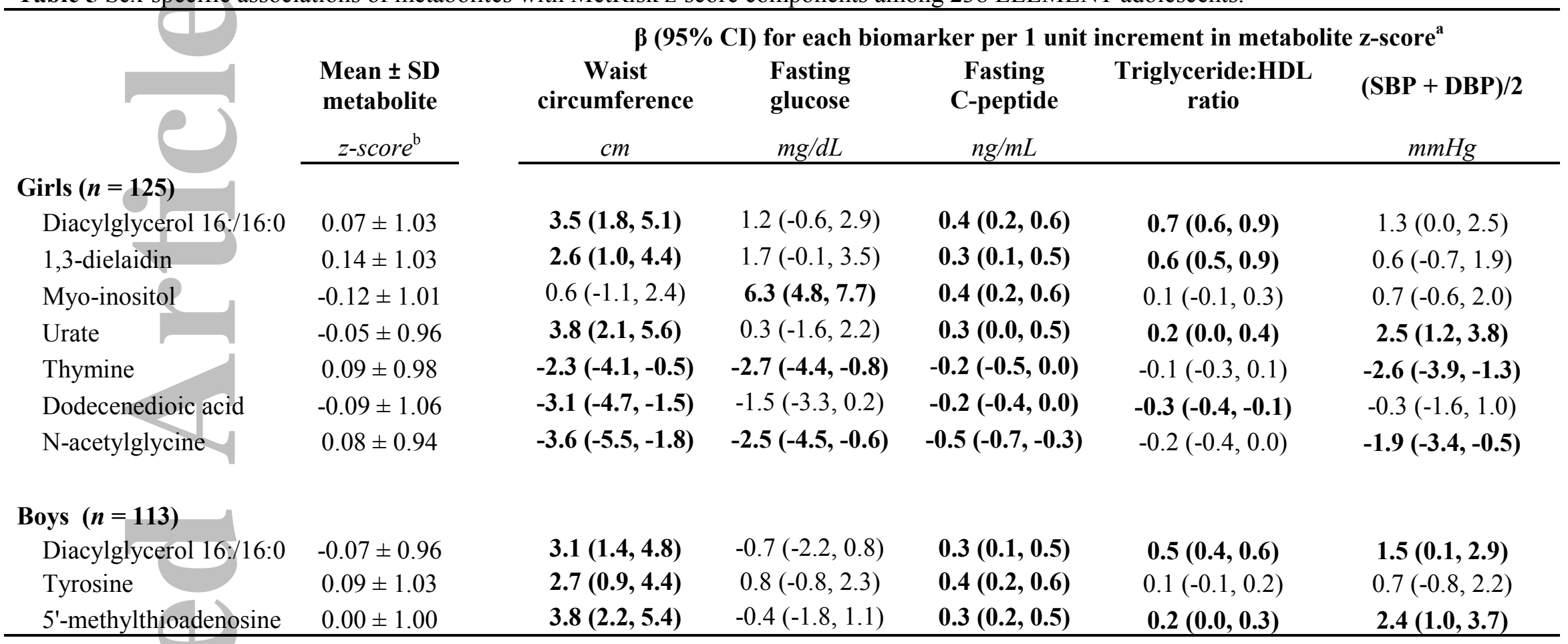

a Adjusted for maternal education, and child's sex and pubertal status.

Bolded estimates indicate statistical significance at $P<0.05$.

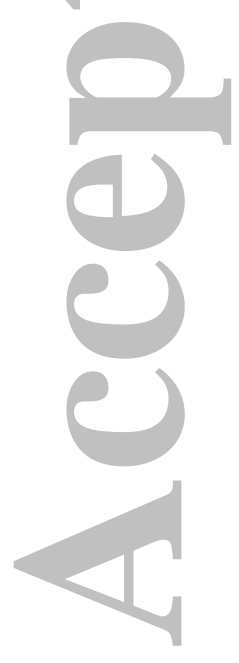


Table 6 Sex-specific associations of branched chain amino acids (BCAA) with MetRisk z-score nad its components among 238 ELEMENT adolescents.

\begin{tabular}{|c|c|c|c|c|c|c|c|}
\hline \multirow{2}{*}{ 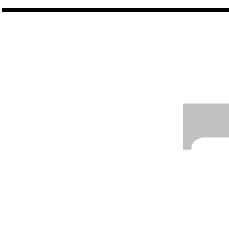 } & \multicolumn{7}{|c|}{$\beta\left(95 \%\right.$ CI) for each biomarker per 1 unit increment in metabolite $z$-score ${ }^{a}$} \\
\hline & $\begin{array}{c}\text { Mean } \pm \text { SD } \\
\text { metabolite } \\
z \text {-score }\end{array}$ & $\begin{array}{c}\text { Waist } \\
\text { circumference } \\
\mathrm{cm} \\
\end{array}$ & $\begin{array}{c}\text { Fasting } \\
\text { glucose } \\
m g / d L \\
\end{array}$ & $\begin{array}{c}\text { Fasting } \\
\text { C-peptide } \\
n g / m L \\
\end{array}$ & $\begin{array}{c}\text { Triglyceride:HDL } \\
\text { ratio }\end{array}$ & $\begin{array}{l}\mathbf{( S B P}+ \\
\mathbf{D B P}) / 2 \\
m m H g\end{array}$ & MetRisk z-score \\
\hline \multicolumn{8}{|c|}{ Girls $(n=125)$} \\
\hline Leucine & $0.04 \pm 0.91$ & $0.0(-2.1,2.2)$ & $-1.1(-3.2,1.0)$ & $0.0(-0.2,0.2)$ & $0.1(-0.1,0.3)$ & $-0.1(-1.7,1.4)$ & $-0.01(-0.13,0.11)$ \\
\hline Isoleucine & $-0.02 \pm 0.93$ & $0.9(-1.2,3.0)$ & $0.0(-2.0,2.1)$ & $0.1(-0.2,0.3)$ & $0.2(0.0,0.4)$ & $-0.3(-1.8,1.2)$ & $0.05(-0.06,0.16)$ \\
\hline Valine & $0.06 \pm 0.93$ & $1.3(-0.7,3.4)$ & $-0.5(-2.5,1.6)$ & $0.1(-0.1,0.4)$ & $0.3(0.1,0.5)$ & $-0.1(-1.6,1.4)$ & $0.09(-0.03,0.20)$ \\
\hline \multicolumn{8}{|c|}{ Boys $(n=113)$} \\
\hline Leucine & $-0.01 \pm 1.09$ & $3.0(1.4,4.5)$ & $-0.8(-2.2,0.6)$ & $0.3(0.2,0.5)$ & $0.0(-0.1,0.2)$ & $1.2(-0.1,2.6)$ & $0.13(0.03,0.24)$ \\
\hline Isoleucine & $0.04 \pm 1.08$ & $3.1(1.5,4.8)$ & $-1.3(-2.7,0.2)$ & $0.2(0.0,0.4)$ & $0.0(-0.1,0.2)$ & $0.8(-0.6,2.2)$ & $0.10(-0.01,0.21)$ \\
\hline Valine & $-0.07 \pm 1.07$ & $2.4(0.7,4.0)$ & $-0.8(-2.2,0.7)$ & $0.3(0.1,0.5)$ & $0.1(-0.1,0.2)$ & $1.4(-0.1,1.9)$ & $0.14(0.03,0.24)$ \\
\hline
\end{tabular}

a Adjusted for maternal education, and child's sex and pubertal status.

Bolded estimates indicate statistical significance at $\mathrm{P}<0.05$.

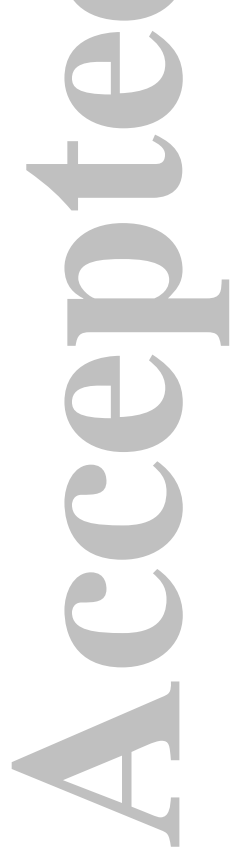


SUPPLEMENTAL MATERIAL: Metabolomic determinants of metabolic risk in Mexican adolescents

Wei Perng ${ }^{1,2}$, Emily C. Hector ${ }^{3}$, Peter X.K. Song ${ }^{3}$, Martha Maria Tellez Rojo ${ }^{4}$, Sasha Raskind ${ }^{5}$, Maureen Kachman ${ }^{5}$, Alejandra Cantoral $^{4}$, Charles F. Burant ${ }^{1,5,6}$, and Karen E. Peterson ${ }^{1,7}$

${ }^{1}$ Department of Nutritional Sciences, University of Michigan School of Public Health, Ann Arbor, MI, USA.

${ }^{2}$ Department of Epidemiology, University of Michigan School of Public Health, Ann Arbor, MI, USA.

${ }^{3}$ Department of Biostatistics, University of Michigan School of Public Health, Ann Arbor, MI, USA.

${ }^{4}$ Center for Research on Nutrition and Health, National Institute of Public Health, Cuernavaca, Morelos, México.

${ }^{5}$ Michigan Regional Comprehensive Metabolomics Resource Core, University of Michigan Medical School, Ann Arbor, MI, USA.

${ }^{6}$ Center for Computational Medicine and Bioinformatics, University of Michigan Medical School, Ann Arbor, MI, USA.

${ }^{7}$ Center for Human Growth and Development, University of Michigan, Ann Arbor, MI, USA.

\section{Correspondence:}

Wei Perng (perngwei@umich.edu)

1415 Washington Heights, Room 1860 SPH 1

Ann Arbor, MI 48109-2029 
Supplemental Table S1 Spearman correlations among components of MetRisk z-score

\begin{tabular}{rccccc}
\hline & $\begin{array}{c}\text { Waist } \\
\text { circumference }\end{array}$ & Glucose & C-peptide & Triglyceride:HDL & (SBP+DBP)/2 \\
\cline { 2 - 6 } Waist circumference & 1.00 & & & & \\
Glucose & 0.02 & 1.00 & & & \\
C-peptide & 0.60 & 0.17 & 1.00 & 1.00 & 1.00 \\
Triglyceride:HDL & 0.39 & 0.02 & 0.40 & 0.18 & \\
(SBP + DBP)/2 & 0.53 & -0.02 & 0.36 & & \\
\hline
\end{tabular}

SBP - systolic blood pressure; DBP - diastolic blood pressure; HDL - high-density lipoprotein
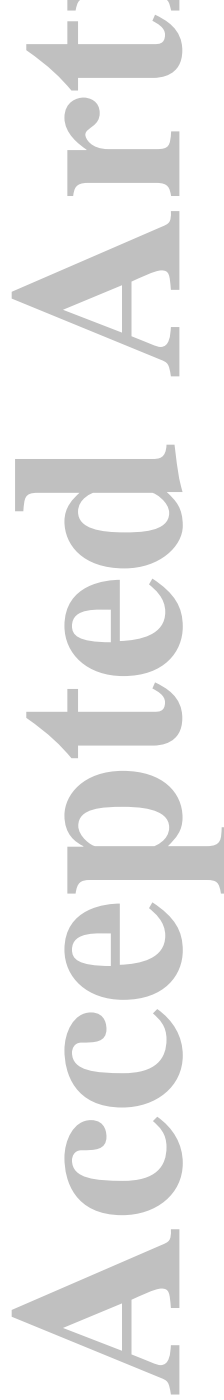
Supplemental Table S2 Sex-specific associations of metabolites with pubertal status components among 238 ELEMENT adolescents. ${ }^{a}$

\begin{tabular}{|c|c|c|c|c|}
\hline & $\begin{array}{c}\text { Pre-pubertal } \\
\mathrm{cm} \\
\end{array}$ & $\begin{array}{c}\text { Pubertal } \\
m g / d L \\
\end{array}$ & $\begin{array}{c}\boldsymbol{\beta}(95 \% \text { CI }) \\
\text { Pubertal vs. } \\
\text { Pre-pubertal } \\
\quad n g / m L \\
\end{array}$ & $P$-value \\
\hline \multicolumn{5}{|l|}{ Girls $(n=125)$} \\
\hline \multicolumn{5}{|c|}{ Metabolites identified via data-driven approach } \\
\hline Diacylglycerol 16:/16:0 & $-0.05 \pm 1.08$ & $0.32 \pm 0.89$ & $0.37(0.00,0.75)$ & 0.05 \\
\hline 1,3-dielaidin & $0.09 \pm 0.98$ & $0.25 \pm 1.11$ & $0.16(-0.22,0.53)$ & 0.42 \\
\hline Myo-inositol & $-0.18 \pm 1.091$ & $-0.01 \pm 1.02$ & $0.17(-0.20,0.54)$ & 0.37 \\
\hline Urate & $-0.16 \pm 0.96$ & $0.17 \pm 0.94$ & $0.33(-0.02,0.68)$ & 0.06 \\
\hline Thymine & $0.01 \pm 1.04$ & $0.24 \pm 0.81$ & $0.23(-0.12,0.58)$ & 0.21 \\
\hline Dodecenedioic acid & $-0.12 \pm 0.99$ & $-0.05 \pm 1.19$ & $0.06(-0.32,0.46)$ & 0.72 \\
\hline $\mathrm{N}$-acetylglycine & $-0.07 \pm 0.95$ & $0.37 \pm 0.86$ & $0.44(0.10,0.78)$ & 0.01 \\
\hline \multicolumn{5}{|l|}{ Branched chain amino acids } \\
\hline Leucine & $-0.02 \pm 0.93$ & $0.15 \pm 0.88$ & $0.18(-0.16,0.51)$ & 0.30 \\
\hline Isoleucine & $-0.15 \pm 0.94$ & $0.24 \pm 0.87$ & $0.39(0.05,0.73)$ & 0.02 \\
\hline Valine & $-0.03 \pm 1.00$ & $0.23 \pm 0.78$ & $0.26(-0.08,0.60)$ & 0.13 \\
\hline \multicolumn{5}{|l|}{ Boys $(n=113)$} \\
\hline \multicolumn{5}{|c|}{ Metabolites identified via data-driven approach } \\
\hline Diacylglycerol 16:/16:0 & $-0.16 \pm 1.04$ & $0.03 \pm 0.89$ & $0.19(-0.17,0.55)$ & 0.30 \\
\hline Tyrosine & $0.10 \pm 1.06$ & $0.12 \pm 0.97$ & $0.03(-0.35,0.41)$ & 0.89 \\
\hline 5'-methylthioadenosine & $0.21 \pm 0.87$ & $-0.15 \pm 1.07$ & $-0.35(-0.72,0.01)$ & 0.06 \\
\hline \multicolumn{5}{|l|}{ Branched chain amino acids } \\
\hline Leucine & $-0.13 \pm 1.01$ & $0.13 \pm 1.15$ & $0.26(-0.14,0.66)$ & 0.21 \\
\hline Isoleucine & $-0.03 \pm 1.05$ & $0.15 \pm 1.07$ & $0.18(-0.21,0.58)$ & 0.37 \\
\hline Valine & $-0.20 \pm 0.96$ & $0.12 \pm 1.13$ & $0.32(-0.07,0.71)$ & 0.11 \\
\hline
\end{tabular}

a Puberty was defined as Tanner stage 2-5 (vs. 1) for breast (girls), testicular (boys), and pubic hair (both) development. 